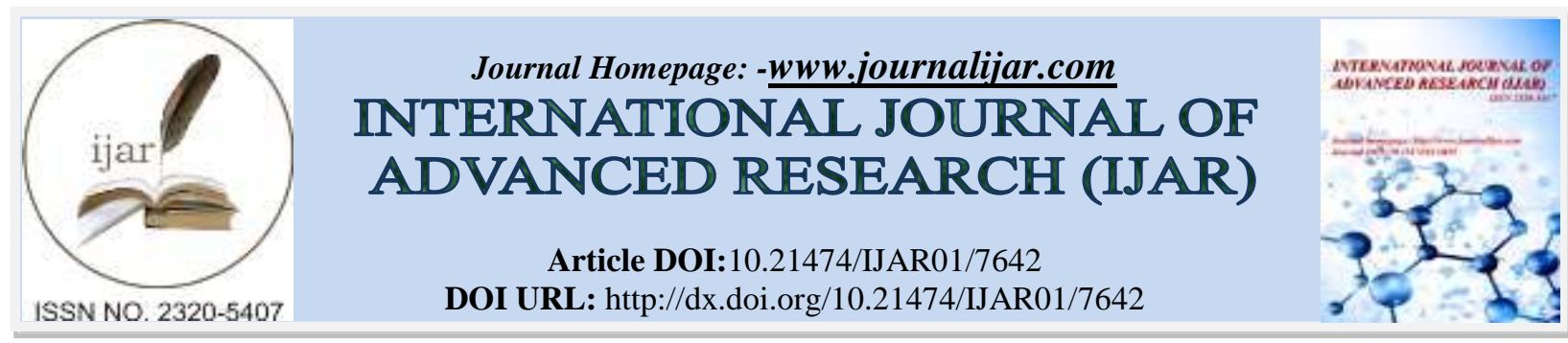

RESEARCH ARTICLE

\title{
NOISE REDUCTION IN MEDICAL IMAGES USING ADAPTIVE WEIGHTED MEDIAN FILTER BASED ON BACK PROPAGATION NEURAL NETWORK.
}

Umamaheswari. J.

\section{Manuscript Info}

Manuscript History

Received: 02 July 2018

Final Accepted: 04 August 2018

Published: September 2018

Keywords:-

Adaptive Weighted Median Filter, Back

Propagation Neural Network, Computed

Tomography images, Noise Reduction,

Liver Cancer.

\begin{abstract}
A novel Adaptive Weighted Median Filter Based on Back Propagation Neural Network (AWM/BPNN) filter is proposed for reducing noise in medical images and improving the performance of median-based filters. The proposed filter achieves its effect through the linear combinations of the median based filters and neural network technique. In this proposed system is a three-stage process. In the first stage, the adaptive technique is used to determine whether the pixel is corrupted or uncorrupted. In the second stage, the weights of the Weighted Median (WM) filter are calculated by using Back Propagation Neural Network (BPNN) algorithm. In the final stage, the corrupted pixel value is replaced by the weighted median value. The visual and performance metrics show that the proposed filter outperforms many of the standard median filters in terms of noise removal with edge preservation.
\end{abstract}

Copy Right, IJAR, 2018,. All rights reserved.

\section{Introduction:-}

The advent of Computed Tomography (CT) imaging, it has been used at an ever-increasing rate and has been established as one of the most significant techniques in the field of medical diagnostic technology. However, The CT images suffer from a type of quantum noise that represents one of the major sources of image quality degradation [1]. Median filtering technique is a nonlinear filtering as opposite to linear image filtering that has control on image noise at the same time as on valuable information. It can eliminate a noise without destroy to a primary information. Median filters are used to remove noise as well as to conserve sharp edges in image efficiently. The median filter is extremely attractive, because it sorts pixels considering brightness in order to get pixel value after filtering. In input image, pixel values are not similar to their neighbors. Great differences between neighboring pixel values indicate a high probability of noise existing in the image [2].

A Weighted median filter is an extension of the median filter. It introduces the concept of weight coefficient into the median filter. Weighted median filters are used to reduce impulsive noise and to preserve the sharp edges in image signal efficiently [3]. This paper aims to design and utilize a method for determining proper weight coefficients based on supervised feed-forward neural networks which use back-propagation learning techniques.

In the proposed algorithm, the noisy pixel is distinguished then replaced with the weighted median value and leaving the remainder image pixel values unchanged. The weights of the WM filter are generated by using BPNN algorithm [4]. The objective of the system is to suppress the noise while preserving image details. The performance of the proposed filter is measured with metric values. 


\section{Principles Of Proposed Algorithm:-}

The proposed algorithm is a decision-based algorithm. It first detects the noisy pixel based on the absolute difference between the pixel values to their neighbors by using the adaptive method. If it has a big difference, that is indicated as a noisy pixel [5]. The Back Propagation Neural Network (BPNN) helps to determine the weight of coefficients for the weighted median filter. Each neuron in the BPNN used to configure a pixel in the image. The operation of the neuron represents the binary weight median filter. On the correction stage, the corrupted pixel is replaced by the weighted median value in the $3 \mathrm{X} 3$ filtering window [6]. This process is repeated for the entire image.

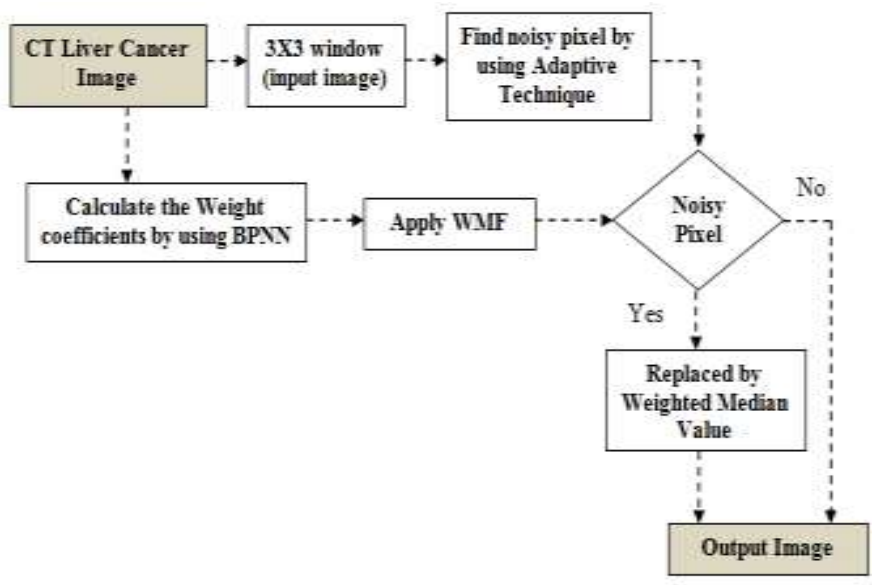

Fig.1:-Outline of proposed methodology

Determine the Weight coefficients by using BPNN:-

BPNN is a supervised learning system as well it is a generalization of the delta rule. It can calculate the desired output for any input in the training set. The vector value of an image is an input to the Back Propagation Neural Network. The BPNN gives the weighted coefficients of an image [7]. In the BPNN system, image structure with three layers of neurons as is illustrated in Fig 2.

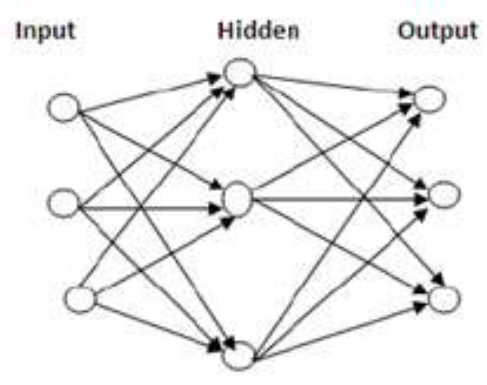

Fig.2:-BPN Network

The number of the neurons of the first layer is equal to the projection data which we get from an original image. The next layer is the hidden layer and has a same number of neurons to the pixels of the image. The number of neurons of the output layer is the same as that of the input layer.

During the training phase, the training data is fed into the input layer. The data is propagated to the hidden layer and then to the output layer. This is called the forward pass of the back propagation algorithm [7]. In further pass, every node in hidden layer obtains input from each the nodes from input layer that are multiplied by appropriate weights and then summed. The output of the hidden node is the nonlinear transformation of the resulting sum. The output values of the output layer are measure up to with the target output values. The target output values are those that try to teach the network. The weight matrix is verified by the method Perceptron Delta Rule, and the projection data can be calculated from the second layer, and inputted to the third layer [12]. 
A common method of training a neural network in which the initial system output is compared to the desired output and the system is adjusted by the difference between the two is minimized. In a back propagation neural network, each neuron adjusts its weights according to what output was expected of it, and the output it gave. This can be mathematically expressed by the Perceptron Delta Rule [13]:

$$
\begin{aligned}
& \Delta \mathrm{w}_{\mathrm{i}}=\mathrm{x}_{\mathrm{i}} \delta \\
& \text { where } \delta=(\text { desire doutput })-(\text { actual output })
\end{aligned}
$$

Remember that $\mathrm{w}_{\mathrm{i}}$ is the array of weights and $\mathrm{x}_{\mathrm{i}}$ is the array of inputs.

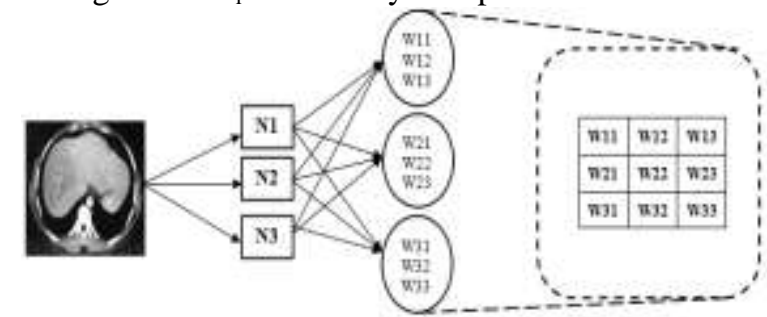

Fig.3:-Methodology of determine the weight coefficients

Calculate the weighted Median Value:-

This method can choose to utilize a weighted median filter with longer window like $2 \mathrm{~N}+1$ to suppress noise efficiently and at the same time protect details lasting less than $\mathrm{N}+1$ points [8]. The general weighted median filter structure is as follows,

$$
\begin{gathered}
\mathrm{X}=\left[\mathrm{X} 1, \mathrm{X} 2, \mathrm{X} 3 \ldots \ldots \ldots \mathrm{X}_{\mathrm{n}}\right] \\
\mathrm{W}=\left[\mathrm{W} 1, \mathrm{~W} 2, \mathrm{~W} 3 \ldots \ldots . \mathrm{W}_{\mathrm{n}]}\right. \\
\mathrm{WM}=\mathrm{MED}\left[\mathrm{W} 1 * \mathrm{X} 1, \mathrm{~W} 2 * \mathrm{X} 2, \mathrm{~W} 3 * \mathrm{X} 3 \ldots \ldots \ldots \mathrm{W}_{\mathrm{n}} * \mathrm{X}_{\mathrm{n}}\right]
\end{gathered}
$$

' $\mathrm{X}$ ' is the input values form an input image, ' $\mathrm{W}$ ' weighted coefficients from BPNN and 'WM' is the weighted median value [11].

\section{Replace the noisy pixel by weighted median value:-}

The adaptive method has been applied widely for performs spatial processing to determine which pixels in an image have been affected by noise. It classifies pixels as noise by comparing each pixel in the image to its surrounding neighbor pixels. A pixel, which is different from a greater part of its neighbor pixels and being not structurally aligned with those pixels to that it is similar, is labeled as noisy pixel. The adaptive process works on a sub-block $\left(S_{\mathrm{xy}}\right.$ ) of the input image. It changes the size of $\mathrm{S}_{\mathrm{xy}}$ during the filtering operation based on certain conditions [9]. The following notation is used:-

$$
\begin{aligned}
\mathrm{Z}_{\min }= & \text { minimum pixel value in } \mathrm{S}_{\mathrm{xy}} \\
\mathrm{Z}_{\text {max }}= & \text { maximum pixel value in } \mathrm{S}_{\mathrm{xy}} \\
\mathrm{Z}_{\text {med }}= & \text { median pixel value from weighted } \\
& \text { median filter }(\mathrm{WM}) \\
\mathrm{xy}= & \text { pixel value at coordinates }(\mathrm{x}, \mathrm{y}) \\
\mathrm{S}_{\max }= & \text { maximum allowed size of } \mathrm{S}_{\mathrm{xy}}
\end{aligned}
$$

The adaptive median filtering algorithm works in two levels. We can denote it by level A and level B as follow [9]:

\section{Level A:-}

IF $\left(Z_{\min }<Z_{\text {med }}<Z_{\text {max }}\right)$ then

$Z_{\text {med }}$ is not a noise go to level $B$ to test if $Z_{x y}$ is a noise

ELSEZ $_{\text {med }}$ is a noise 
1. Increase the window size

2. Repeat Level A until...

$\mathrm{Zmed}$ is not a noise

Level B:-

IF $\left(Z_{\text {min }}<Z_{x y}<Z_{\text {max }}\right)$ then

$\mathrm{Z}_{\mathrm{xy}}$ is not a noise

Output is Zxy

ELSEZ $_{\mathrm{xy}}$ is a noise

Output is WM

\section{Proposed Algorithm Description:-}

The proposed algorithm is basically a two stage algorithm in which the first stage is the noise detection and the second stage is noise removal. The main stages of the proposed algorithm to noise removal is as follows,

Step 1: Get the noisy image as X.

Step 2: Calculate weight matrix form input image $X(i, j)$ as $W(i, j)=w_{1}, w_{2}, \ldots, w_{n}$ by using the method Perceptron Delta Rule in BPNN.

Step 3: Using the above weights to calculate weighted median value as follows,

1. If the selected window, ranges from $X=\left[\mathrm{x}_{1}, \mathrm{x}_{2} \ldots \mathrm{x}_{\mathrm{n}}\right], \mathrm{W}=\left[\mathrm{w}_{1}, \mathrm{w} 2 \ldots \mathrm{w}_{\mathrm{n}}\right]$.

2. $\mathrm{WM}=\operatorname{Med}\left[\mathrm{w} 1 * \mathrm{x} 1, \mathrm{w} 2 * \mathrm{x} 2, \mathrm{w} 3 * \mathrm{x} 3 \ldots \ldots . . \mathrm{w}_{\mathrm{n}} * \mathrm{x}_{\mathrm{n}}\right]$

Step 4: Calculate the number of detected noisy pixels per $\mathrm{N} \times \mathrm{N}$ window by detecting noisy pixel using $3 \times 3,5 \times 5$ and $7 \times 7$ windows on input image $X$.

Step 5:Sort all the pixels $\mathrm{NxN}$ sub window ' $\mathrm{sw}$ ' from original image $X(\mathrm{i}, \mathrm{j})$. Find the med value of 'sw'. It frequently increases the 'sw' size by comparing median value with extreme values of image. However, the biggest intensity differences are assigned to corrupted pixels by noise.

Step 6: Determines the corrupted and uncorrupted pixels adaptively as per the above step. If it is a corrupted pixel then its value is replaced by weighted median value 'WM'

Step 7: If it is an uncorrupted pixel and its value is left unchanged to preserve the edge and other significant details in medical image.

Step 8: The process is done iteratively.In this paper, we propose a design of the adaptive weighted median filter, based on the Perceptron Delta Rule by using a back-propagation algorithm.

\section{Evaluation Parameters And Experimental Results:-}

In order to test whether the proposed algorithm in this paper can effectively filter the medical image noise with different densities and reserve the image details, some experiments are carried out on the platform of Matlab7.0 by the liver cancer CT images with size of $256 \times 256$. With this experimental result, we have made a comparison along with the proposed filter by the standard median filter, weighted median (WM) filter and adaptive median (AM) filter in that our method proves to be greater. Objective results are shown for all filters as follows, 


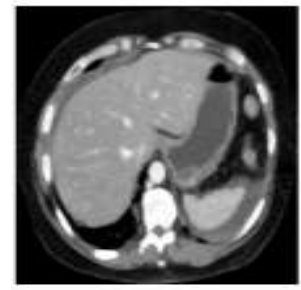

(a) Median

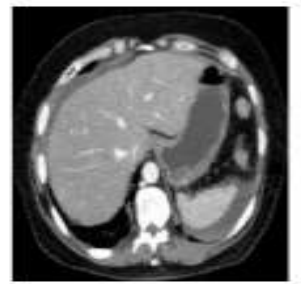

(c) Adaptive Median

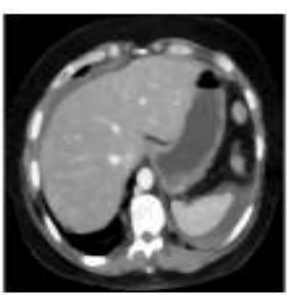

(b) Weighted Median

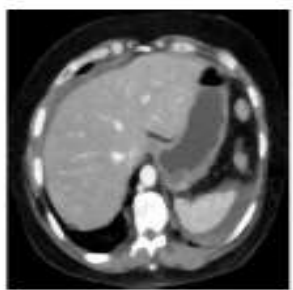

(d) AWMBPNY

Fig4:-Results of Median based filters on CT image affected by noise.

From the above results it is found that the proposed filter is outperforming in denoising procedures without losing the useful information such as edges and textures. In Fig 4, (a) represents the median, the second image (b) represents the WM, the third (c) represents the AM and the fourth (d) represents our proposed method. It is evident from these figures that the above denoised images using our proposed method have better visual quality than that using other median based filters.

Table 1:-Comparison of Results of WM, AM, CWM and HE/ACWM filters

\begin{tabular}{|l|c|c|c|c|}
\hline & Median & Weighted Median & Adaptive Median & AWMBPNX \\
\hline SUR & 28.0821 & 28.9937 & 32.3692 & 40.1108 \\
\hline RUISE & 89554 & 7.2114 & 2.4401 & 0.9273 \\
\hline MAE & 125673 & 11.9337 & 2.7600 & 0.9875 \\
\hline SSLI & 0.9625 & 0.8664 & 0.9474 & 0.9853 \\
\hline
\end{tabular}

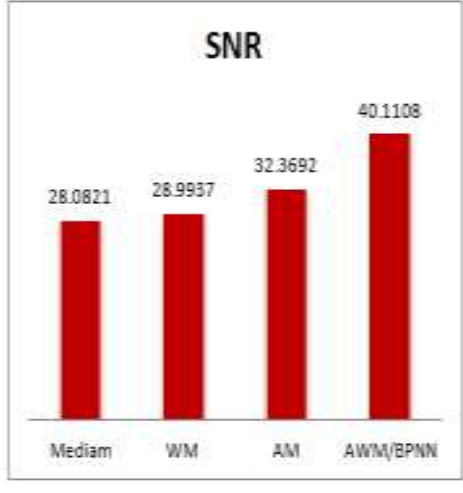

(a)

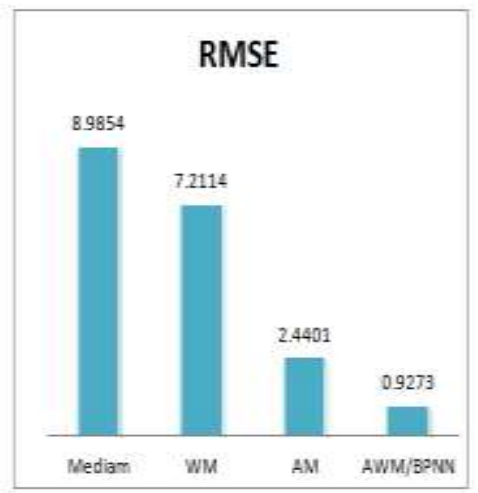

(b) 

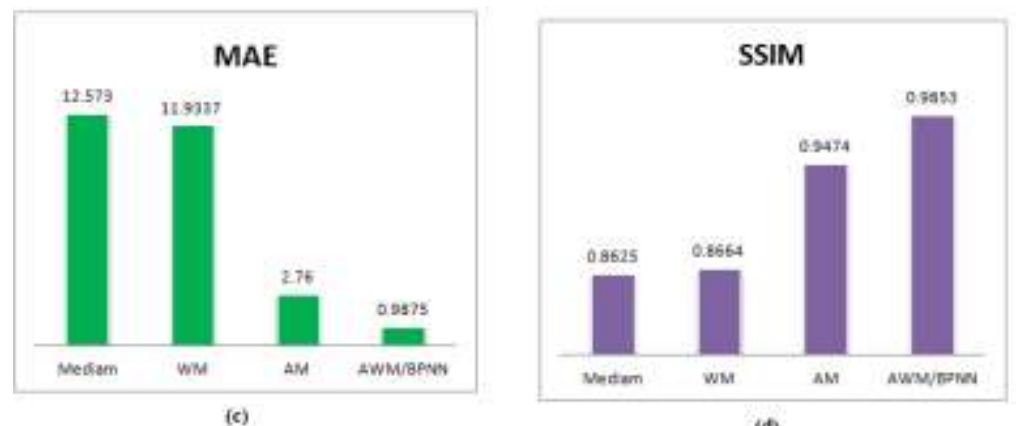

Fig5:-Performance elevation metrics for Median, WM, AM and AWM/BPNN filters.

In Table 1, results shown that the SNR,SSIM gains and RMSE,MAE decreases to CT scan images for our proposed technique [14] [15]. The above results and analysis illustrates that the proposed method outperforms over the existing median based filter methods.

A median filter among conventional methods performs well for low-density noise but for higher densities, it provides artifacts along edges. AMF and AWMF fail to remove noise, whereas SAMF [16] [17] restore all noisy pixels but blurring edges of medical image. The proposed method not only preserves edges for low density of noise but also performs very well for noise density as high as $90 \%$. Existing method totally fails to protect regions which have pure white or pure black background. since pure white or pure black regions give confusion as they have same intensity as of the impulse noise but the proposed method, irrelevant of intensity values and provides edge as well as detail preservation in all cases.

\section{Conclusion:-}

Denoising of images is a major task in medical image processing system. In this paper, a new method for getting the weight coefficients based BPNN techniques has been designed and implemented. Adaptive weighted median filter based neural network shows efficient noise suppression and an excellent image detail-preserving capability for liver cancer CT scan image. The effectiveness of the proposed method is demonstrated by the experimental results. The proposed filter can eliminate the noise without deteriorating the original image. Experimental results show the proposed method can improve the filtering performance significantly.

\section{Reference:-}

1. Kalra MK, Naz N, Rizzo SM, Blake MA. Computed tomography radiation dose optimization: scanning protocols and clinical applications of automatic exposure control. Curr Probl Diagn Radiol 2005; 34:171-181.

2. Shanmugavadivu P and Eliahim Jeevaraj P S "Adaptive Pde-Based Median Filter For The Restoration Of HighDensity Impulse Noise Corrupted Images” International Journal of Advanced Information Technology (IJAIT) Vol. 1, No.6, December 2011.

3. http://www.generation5.org/content/2002/bp.asp

4. J. Kowalski, T. Kacprzak "Cellular neural network based weighted median filter for real time image processing" Proceedings 2001 International Conference on Image Processing, ISBN: 0-7803-6725-1.

5. Feras N. Hasoon, Jabar H. Yousif, Nebras N.Hasson and Abd Rahman Ramli "Image Enhancement Using Nonlinear Filtering Based Neural Network” Journal Of Computing, May 2011.

6. Fry.J, Pusateri. M, "High Speed Pipelined Architecture for Adaptive Median Filter," Applied Imagery Pattern Recognition Workshop (AIPR), 2010, IEEE.

7. Asmatullah,anwar.M.Mirza,and asifullah Khan," Blind Image Restoration using Multilayer Back Propagation", Proceedings of the International Multi-topic (INMIC 2003),IEEE Conference,Islamabad,pp.55-58,December 2003.

8. LI Lin-lin,WANG Chang-you,YANG Fu-ping,GONG Hui "A new kind of adaptive weighted median filter algorithm" 2010 International Conference on Computer Application and System Modeling (ICCASM 2010).

9. O. Um a Maheshwari, G.B. Vanisree, Dr. D. Ebeneze $r$ "PERFORMANCE OF SEVERAL TYPES OF MEDIAN FILTERS IN SPECTRAL DOMAIN" springer,Intelligent Information Processing II ,2017.

10. Feras N. Hasoon, Jabar H. Yousif, Nebras N.Hasson and Abd Rahman Ramli “ Image Enhancement Using Nonlinear Filtering Based Neural Network” Journal Of Computing, May 2011. 
11. Suganya C., Dr. O. Umamaheswari,'Image Restoration Using Noise Adaptive Fuzzy Switching Weighted Median Filter for the Removal of Impulse Noise"IEEE.

12. Mitsuji Muneyasu, Taltahiro Mae\& a,nd Taltao Hinainoto "A New Realization of Adaptive Weighted Median Filters Using Counter Propagation Networks" 1999 IEEE.

13. Montreal, Canada, "Pattern classification by Assembling small Neural networks" IEEE Proceedings of International Joint conference on Neural networks, july 31-August4, 2005.

14. Z. Wang, A.C. Bovik, H.R. Sheikh, E.P. Simoncelli, Image quality assessment: from error visibility to structural similarity, IEEE Transaction on Image Processing vol. 13, No. 4 , April 2004, pp.600-611.

15. Debdoot Sheet, Santanu ParI, Arindam Chakraborty, Jyotirmoy Chatterjee and Ajoy K. Ray t "Visual Importance Pooling for Image Quality Assessment of Despeckle Filters in Optical Coherence Tomography" 2010, IEEE.

16. V.S. Bhadouria, D. Ghoshal, A.H. Siddiqui, A new approach for high density saturated impulse noise removal using decision-based coupled window median filter. SIViP 8(1), 71-84 (2014).

17. H.M.R. Afzal, J. Yu, Y. Kang, Impulse noise removal using fuzzy logics. IEEE Annual Academic Conference of Chinese Association of Automation 32, 413-418 (2017). 